\title{
The breakdown on bioinformatics
}

\begin{abstract}
Hardware and software make up this survey - items include software for genomics work, DNA and protein sequence analysis, protein viewing, and a number of products for drug discovery and drug screening applications.
\end{abstract}

\section{Bioinformatics Workbench \\ From MDL Information Systems \\ An intranet-based 'virtual laboratory' that delivers data and computing tools for genomic research}

Developed at the National Center for Supercomputing Applications (NCSA) at the University of Illinois, the Bioinformatics Workbench provides an environment that integrates the genome-related tools and databases emanating from sequencing efforts on many plant and animal species. This system is designed to tie together software that is already available. Workbench offers a platform-independent interface to more than 100 public domain databases and software packages for DNA and protein sequence analysis. With this software, scientists connect via a Web browser on their corporate intranets to search all major molecular and structural biology databases. The Bioinformatics Workbench uses a generic design that allows tools, databases, servers and computer engines based on different formats to interoperate. The interface to these components works with any standard forms-capable, HTML browser. Previously known as Biology Workbench, the program was developed by the Computational Biology Group of the NCSA. The NCSA version is available free to the academic community at $<$ http://bioweb.ncsa.uiuc.edu>

Reader Enquiry No. 100

\section{LifeTools 3D}

From Incyte Pharmaceuticals

Data mining and visualization software for searching genomic information

The program displays genomic data as interactive, multidimensional graphics. It can present up to five dimensions of data simultaneously, allowing scientists to collate factors, such as gene, tissue type and abundance. Users can go through the threedimensional presentations: rotate, pan and zoom to observe the data; move up or down through layers of information; and change filter settings at any point to expand or narrow the data set. According to Incyte, this visual approach not only makes it easier to investigate the gene sequences in the LifeSeq database, but also allows scientists to spot trends in gene expression and locate novel genes more easily. Incyte has used the program to identify gene fragments in LifeSeq that should be considered for inclusion in the LifeSeq FL database of full-length genes and the LifeSeq Atlas gene mapping database. In

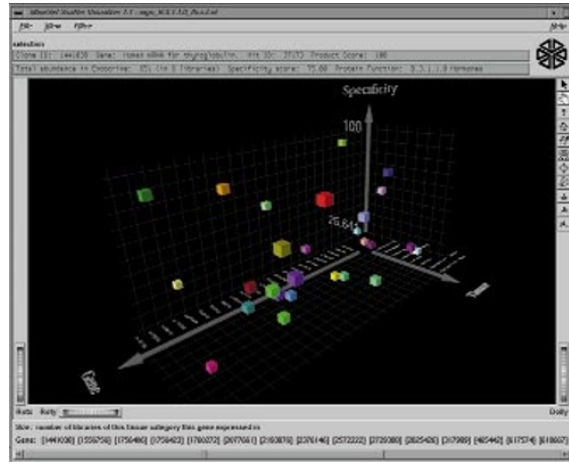

Incyte Pharmaceuticals' LifeTools 3D software.

collaboration with Silicon Graphics, the company has created customized functions that are designed to help explore LifeSeq, including Protein Function Hierarchy Visualization, which displays the relative expression of genes across tissues. There is also MultiGene northern visualization, which uses a threedimensional graph to show the tissue distribution for a selected set of genes. This program is a UNIX-based, client/server application, requiring Mineset 1.1 (offered by the company with LifeTools 3D).

Reader Enquiry No. 101

\section{MM3 Software}

From Tripos

The 1996 version of the company's molecular mechanics software

As with previous versions, this software can be accessed directly from the company's Sybyl suite of molecular design, visualization and analysis software. The company has also announced plans to expand the integration of its Sybyl suite with MM3. The plan is for MM3 to function as an energy server for Sybyl and other Tripos software packages, supplying molecular mechanics energies and force-field gradients on demand. In addition, Tripos is funding the development and testing of methods for MM3 to treat biomolecular structures, such as proteins, peptides and DNA.

Reader Enquiry No. 102

\section{GeneWorld 21}

Pangea Systems

A bioinformatics database application for automated sequence annotation and analysis

This product should find use in informationbased pharmaceutical organizations seeking to use bioinformatics to discover new drug targets. GeneWorld 2.1 uses simplified, userdefinable, automated workflow 'strategies', which enable automated analysis and anno- tation of thousands of sequences. Once defined, these strategies can be saved and used for subsequent analyses, standardizing and distributing bioinformatics information throughout the user's organization. By selecting analysis steps from pull-down menus, customers can define strategies for automated gene finding, domain finding, functional identification, EST analysis and secondary structure prediction. A technology agreement with Paracel enables customers to include accelerated Smith-Waterman, pairwise alignment analyses in their strategies Reader Enquiry No. 103

\section{Gene Explorer}

From MSI

A new computational environment for the analysis of DNA and protein sequences, as well as protein structures

From restriction analysis to virtual sitedirected mutagenesis, Gene Explorer strives to meet the computational needs of molecular biology researchers within a single environment. It should help to focus the user's research on the most promising leads, hypotheses and candidate genes. In a series of steps, 3D Structure Prediction will transform protein sequence into a three-dimensional structure model, search databases for similar solved protein structures, present search results in a graphical display of homology, automatically align sequence homologues and highlight regions of similarity. Furthermore, it invokes Modeler algorithms to generate a model three-dimensional structure, verifying model structure using Profiles-3D technology. The process is presented in a simple, step-by-step interface, which is stated to enable users to build a model in a matter of minutes.

Reader Enquiry No. 104

\section{Jaguar}

From Schrödinger

Version 3.0 of the PS-GVB ab initio package has had its name changed to Jaguar

Jaguar includes a number of performance enhancements, with large speed-ups for density functional theory, as well as a new theoretical method, GVB-LMP2, which should give highly accurate conformational energies of molecules. Numerical methods are said to make this program a fast general-purpose program, which allows it to handle molecular systems previously considered intractable. According to the manufacturer, this package is useful for such operations as viewing electrostatic potentials of the different bind- 
ing pockets of related proteins. The initial release included generalized valence bond (GVB) theory, which incorporated the pseudospectral (PS) numerical methods for fast evaluation of the two-electron integrals. Over the last three years, however, the product has outgrown its name in terms of functionalities and capabilities.

Reader Enquiry No. 105

\section{Hardware systems}

\section{DeCypher II}

From Time Logic

A system designed to provide the

infrastructure to handle large-scale DNA

sequence comparison analysis

The ultra-high performance cluster of this sequence similarity search system should help to identify rapidly similar sequences and deliver results quickly to the geneticist. The system's logic circuit adapts to additional algorithms through software reconfiguration, eliminating hardware obsolescence associated with older technologies. System specifications include 1.5-12 billion affine SW cells per second per rack, 1-8 15-accelerator systems per $48-\mathrm{cm}$ rack, Xilinx reconfigurable computational arrays, 1-8 single- or dual-SMP Pentium CPUs, a Windows-NT TCP/IP network server, cluster scalability and a 32-144 GB hard-drive capacity per rack. CPU clusters offer high Smith-Waterman throughput, starting with a single 15-accelerator system. Each rack expands to hold up to eight systems, for a total of 120 Similarity Engine accelerators at a stated 12 billion affine SW cells per second. Special faulttolerant versions are also available. The company offers custom hardware and software development and support. According to the manufacturer, virtually unlimited scalability is achieved by partitioning the query stream across one or more racks. Each 15-card CPU contributes up to 1,500 million affine SW cells per second. Users can reorganize the cluster for rapid interactive results, while production searches are streamed as a batch-mode, background process as resources become available. A simple, automatic script-based, semaphore mechanism pauses each CPU, permitting periodic database updates to be cloned across the cluster without loss of queued search requests or results. By using standard Intel technology with Windows-NT, the cost of ownership is lowered.

Reader Enquiry No. 106

\section{Octane workstations}

From Silicon Graphics

A new line that features high-performance graphics, symmetric multiprocessing and a pure 64-bit computing environment

Octane workstations are said to have improved systems and I/O bandwidth, giving greater flexibility to expand their applications. The system shares many components with the company's supercomputing architecture, employing a unique seven-port crossbar switch in lieu of the conventional shared bus architecture. It also combines this high-bandwidth switch with single or dual MIPS R10000 processors and fast desktop graphics. Furthermore, it is said to offer a 70 per cent increase in floating-point performance over the Indigo 2 Impact 10000 family. With a peak bandwidth of 1.6 GB s11 per port, the seven-port crossbar switch is stated to provide as much as 210 times the bandwidth found in traditional architectures. The system is designed to process multiple data types, including audio, video, imagery and three-dimensional geometry. With hardware-accelerated geometry processing and high-speed texturing and image processing, Octane systems enable real-time visual imagery for applications ranging from solid modelling, defence imaging, threedimensional animation and emerging solu- tions, such as real-time, three-dimensional volume roaming.

Reader Enquiry No. 107

\section{Moresoftware}

\section{Synergy}

From NetGenics

An alliance with Incyte Pharmaceuticals will link Synergy and Incyte's genomic database software

Synergy assists in the drug discovery process by enhancing the researcher's ability to access and analyse biological information. Incyte will incorporate certain aspects of NetGenics' software and the CORBA (common object request broker architecture) framework into its database products and the companies will jointly develop a product that is designed to provide increased functionality to Incyte's database and software subscribers by facilitating project management and allowing the integration and query of multiple, unrelated relational databases. Synergy is a cross-platform, client/server-based bioinformatics computer software system. It parallels the organization of multidisciplinary drug teams and enables the members of a drug team to communicate efficiently.

Reader Enquiry No. 108

\section{Diva and $\mathbf{R S}^{3}$ Discovery}

From Oxford Molecular

Six products designed to increase the speed and efficiency of the drug discovery process

These software packages add some 25 software tools for use in academic research and the pharmaceutical, chemical and biotechnology industries. Each product - Diva, CAChe 3.0, Tsar 3.0, Topkat 5.0, UniChem 4.0 and RS $^{3}$ Discovery - deals with a different aspect of the process of drug discovery; taken together, they should help scientists predict the properties and toxicities of drug candidates and enable comparisons with

ADVERTISEMENTS

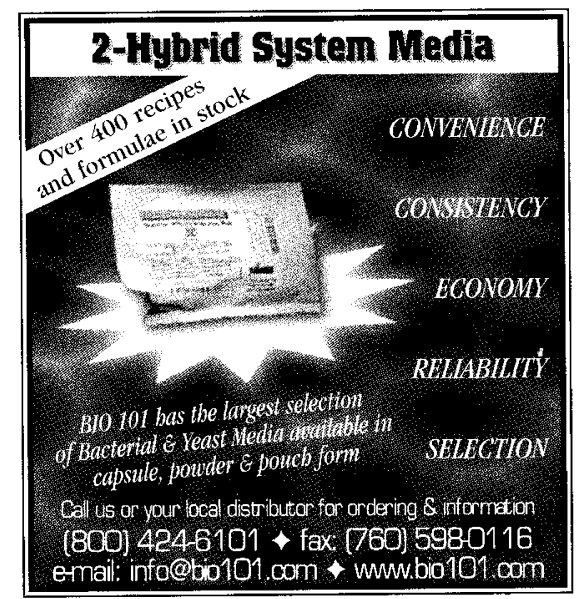

Gramsch Laboratories
Custom Pept/de Synthes/s:
Shipped within one week:
10 AA DM $400.00 ; 15$ AA DM 520.00;
20 AA DM 660.00; $10-30$ mg unpurified;
buy only when satisfied with the HPLC profile
Antiserum Productlon Service:
you prepare the recombinant protein (in paa-gel
or soluble), we produce the antiserum
Distributor for Japan: FUNAKOSHI CO, Tokio 113
Gramsch Laboratories
D-85247 SCHWABHAUSEN, Germany
Phone +49-8138-1769 or +49-8131-5679-0
Fax +49-8138-1260 or +49-8131-5679-24

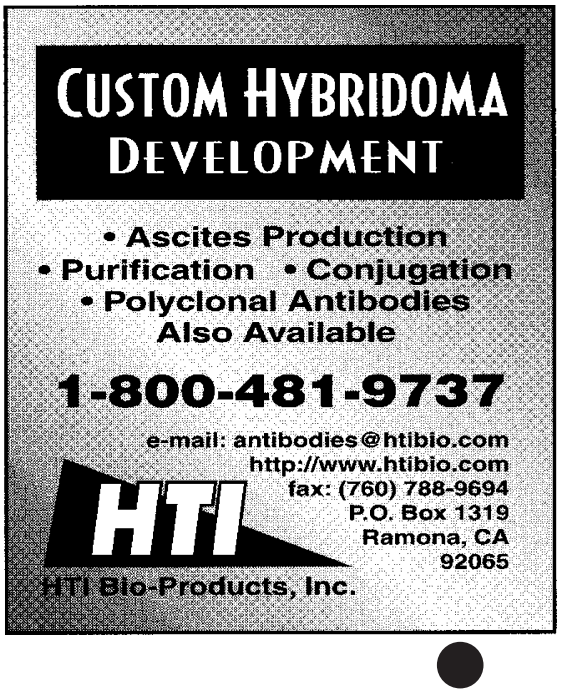


chemicals in existing databases. Diva was created to allow researchers to mine corporate databases and identify the properties and relationships of thousands of compounds. Version 3.0 of CAChe allows experimental chemists to predict and analyse properties of drug candidates and chemicals on a PC. Tsar 3.0 was written to help identify structure-activity relationships quickly by integrating QSAR data, computations and statistics into one package. Topkat, now in version 5.0, offers a Windows environment and focuses experimental testing by quickly and accurately assessing the toxicity of drug candidates based on structure. UniChem optimizes chemical reactions with property predictions that should aid in the design of catalysts and in the prediction of reaction mechanisms. Lastly, RS $^{3}$ Discovery uses Oracle to store and retrieve information in a flexible open architecture.

Reader Enquiry No. 109

\section{extendLRS}

From Genomyx

Software for automated DNA sequencing and analysis

The extendLRS sequencing software has been designed primarily for the analysis of

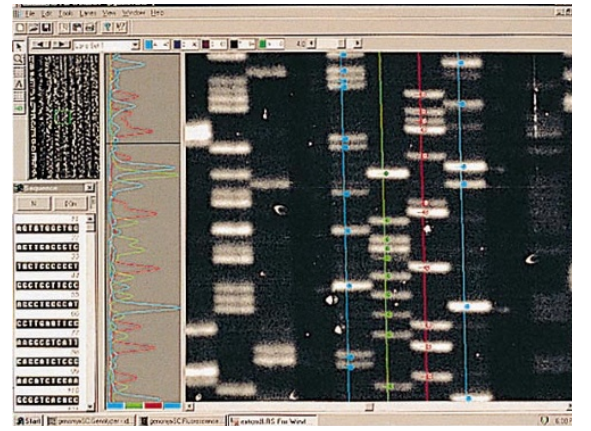

extendLRS sequencing software from Genomyx.

images obtained from the Genomyx SC fluorescent imaging system. It also processes images from other sources, including autorads and images as large as $50 \mathrm{Mb}$. This system includes the company's automatic band detecting and labelling, and lane tracking technology.

By eliminating manual calculations and guesswork, extendLRS increases base-calling accuracy, says Genomyx. The new software can read sequences as long as 400 bases with a high degree of accuracy. Several software tools are included for stepping through and editing the sequence. DNA sequence information can be exported in ASCI text or SCF format for use in other applications - TIFF images may be imported or exported. Images and sequences can be printed on any standard printer as they are or with lanes, band labels and annotation.

Reader Enquiry No. 110

\section{Antisense Functional Genomics}

From Sequitur

The commercial availability of

second-generation antisense compounds

These compounds have been developed through careful screening in a cell culture assay system. With this program, the company does not retain the rights to the research findings derived through their use.

Advantages of these compounds are said to include improved specificity and reduced toxicity, target gene inhibition with only three to six compounds screened, as well as rapid and cost-effective results, according to Sequitur.

Reader Enquiry No. 111

These notes are compiled by Brendan Horton from information provided by the manufacturers. For more details, fill in the reader service card bound inside the journal.

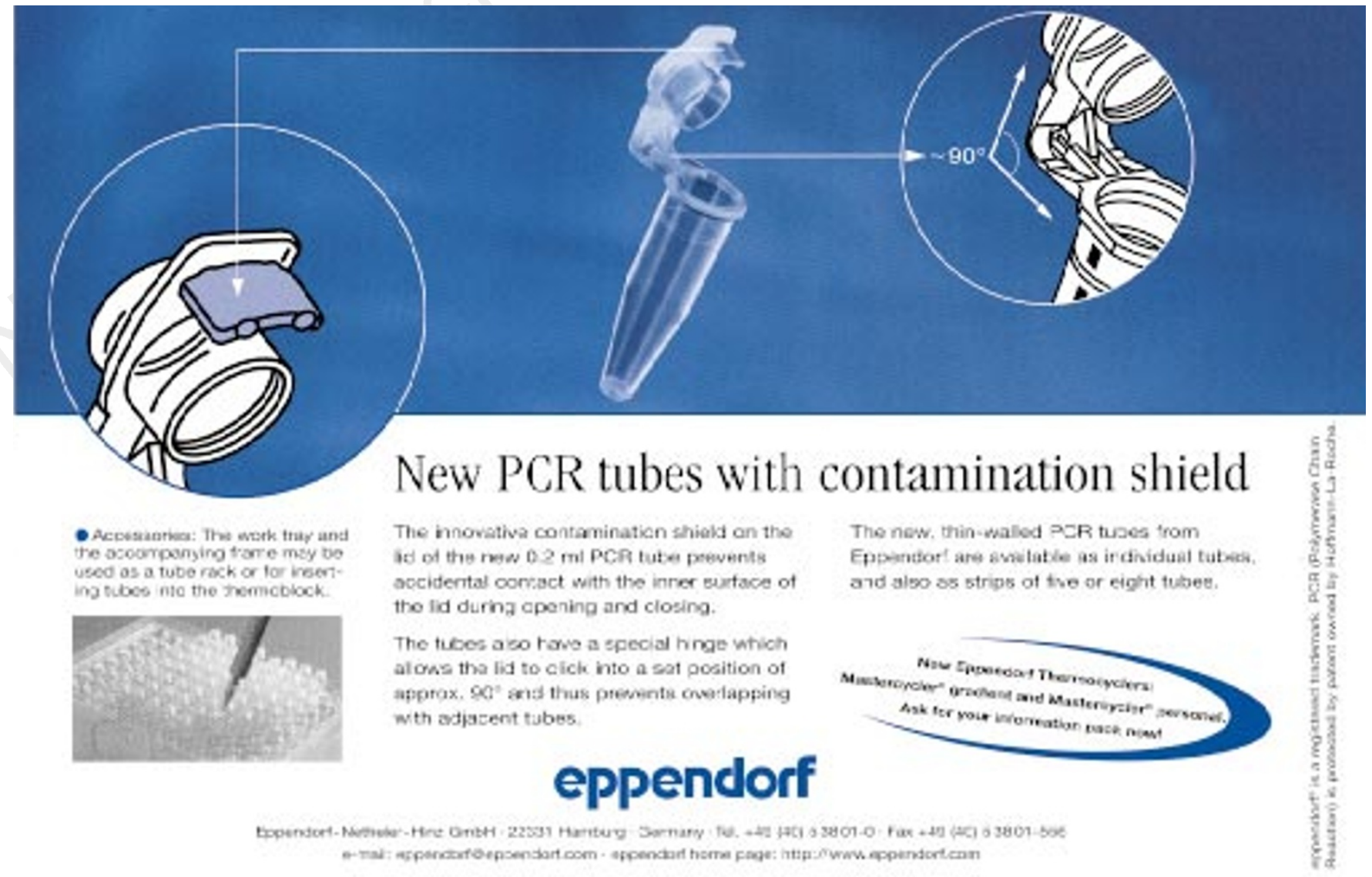

